\title{
Estimating net ideal cycle time for body-in-white production lines
}

\author{
WC Grobler* \\ DJ Kotze ${ }^{\dagger}$ \\ JW Joubert ${ }^{\ddagger}$
}

Received: 3 August 2020; Revised: 2 June 2021; Accepted: 2 June 2021

\begin{abstract}
In the automotive industry, a Body-in-White (BIW) refers to the first step, the basic structure, in the production of a vehicle. Once a BIW production line has been built, the (maximum) capacity is fixed and throughput is therefore limited by the equipment specified during the design phase. The main metric used to inform the production line design is the Net Ideal Cycle Time (NICT). Unfortunately the state of practice to estimate the NICT is a basic heuristic that does not account for production variation. In this paper we challenge the current estimation approach by proposing an alternative that assumes actual production to follow a Weibull distribution. The proposed model is derived and estimated from empirical data. The results suggest that BIW production lines have traditionally been designed with too low a capacity, resulting in planned throughput rarely being achieved. On the other hand, increasing the design capacity implies higher initial investment. In this paper it is demonstrated that the higher investment required is offset by reduced losses, resulting in more reliable planning and returns.
\end{abstract}

Key words: Automotive industry, design of production systems, capacity planning, investment appraisal, cycle time estimation, body-in-white.

\section{Introduction}

The BIW production line in the automotive manufacturing industry is responsible for creating the basic structure of a vehicle. Figure 1 illustrates, through an explosion diagram, the basic structure of the BIW.

The assembly stations along the production line are typically heavily equipped with robotic welders and automated material handling technology. In this paper the term 'BIW' will

${ }^{*}$ Department of Industrial and Systems Engineering, BMW South Africa, Rosslyn, Pretoria, South Africa, email: willem.ga.grobler@gmail.com

${ }^{\dagger}$ Department of Industrial and Systems Engineering, BMW South Africa, Rosslyn, Pretoria, South Africa, email: dirk.j.kotze12@gmail.com

${ }^{\ddagger}$ Corresponding author: Centre for Transport Development, Industrial \& Systems Engineering, University of Pretoria, South Africa, email: johan.joubert@up.ac.za 


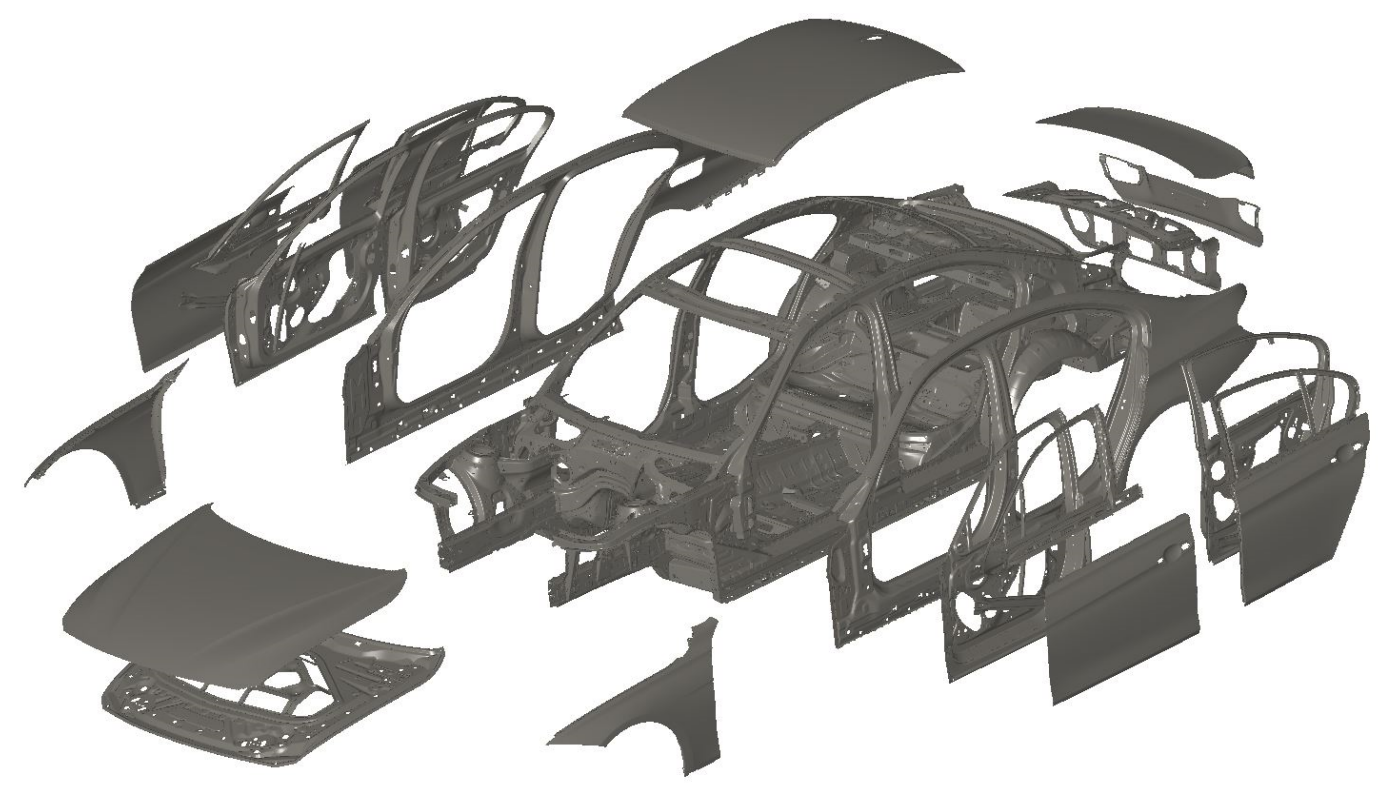

Figure 1: Body-in-White (BIW) structure of 2012 BMW 3 series

mainly refer to the production line and not to the vehicle structure, unless explicitly stated otherwise.

Designing the BIW line is generally a complex and challenging task. The equipment selection is subject to the required throughput and, once installed, essentially becomes the ceiling for production. Production line designers use the NICT as the reference to specify equipment capacity, which, in turn, is informed by two important design parameters. The first is Takt Time (TT), the average time between the start of production of one unit and the start of production for the next, subsequent unit and, secondly, the Overall Equipment Efficiency (OEE).

If the NICT is short, as is the case in a fast production line, then more robots or people are required to complete a process step. When the NICT is longer, fewer robots or people are required and, consequently, results in lower financial investment. It is for this reason that the NICT of a (BIW) production line must be reliable. Too high an estimate results in inefficient use of capital, while too low an estimate means actual production cannot meet the set targets.

In the current literature we distinguish between two streams of cycle time research. Cycle time estimation is the focus of this project and deals with the design or pre-production stages of the line's lifecycle [3]. Cycle time optimisation, on the other hand, deals with analyses of and methods to improve the cycle time of a system that is already in a series-production stage.

Although much research has been done in the semiconductor industry with regards to cycle time estimation, the state of practice in the majority of industries rely on fairly 
basic heuristics. Production variation is not accounted for other than through a scalar, average safety factor like OEE.

This paper contributes to the body of knowledge by proposing a new NICT estimate, one that takes production variation into account. The proposed model is derived from studying empirical production data over a multi-year period in the automotive BIW environment. The analyses suggest that actual production follows a Weibull distribution, where the shape parameter can be estimated from historical data, and the scale parameter is used to estimate the NICT.

We show that the NICT, when estimated using traditional approaches, leads to a production line that consistently suffers from under-performance. Consequently, expensive production losses are incurred. The proposed estimate requires lines to be designed with a slightly higher capacity. Although this implies higher initial investment, we demonstrate in this paper that the increased investment is offset by reduced losses. A direct consequence is that production planning is more realistic, and performance of the lines are more reliable.

The remainder of this paper is arranged as follows. In the next section we review current literature in cycle time estimation and optimisation. In Section 3 we explore the current state-of-practice NICT estimation method, and demonstrate it's limitation for design engineers. In Section 4 we study the throughput distribution of a real BIW production line. We fit an appropriate throughput distribution from empirical data over the 2012-2015 period, and lay the foundation for the proposed NICT estimation. The Weibull-based estimation approach is proposed in Section 5, and in Section 6 a comparative benchmark is done to evaluate the feasibility of the proposed method in terms of costs. The paper is concluded in Section 7 along with a brief research agenda.

\section{Cycle time estimation and optimisation}

Cycle time estimation is important in various industries. There has been a significant amount of research completed within the semi-conductor industry, with popular estimation techniques being statistical modelling [10, 13], heuristics [1, 18, aggregation [14] and historic correlation approaches [2, 5, 7]. The goal of these research contributions are to develop models that could estimate the cycle time of series-production systems. The estimation aims to get a handle on predictable production output, and then compare the system performance against customer demand.

More general production systems have seen fewer research contributions. Xu et al. [15] focus their research on cycle time estimation for unreliable production lines. The research does not state for which industry this specific estimation is valid, but specifies that the type of production lines under investigation are multiple product production lines. The study is related to BIW production lines in terms of the unreliability of the production lines. In their research they use chance constraint and fuzzy linear programming to predict production cycle time so that they can calculate production line efficiency. The main model utilised for this is the one from Johri [8]. Presented in 1987, this linear programming model is used to calculate the capacity of deterministic production lines of various products. 
In Yang et al. [16] the focus of research falls on the relationship between cycle time and production throughput. Again there is no reference to the specific industry. They suggest that the cycle time-throughput percentile of the production system could be used for strategic planning purposes. The main assumption for their research is the use of the generalised gamma distribution to represent actual cycle time. Simulation is then used as an evaluation method to support their theory.

Chen \& Zhou [3] propose quantile regression as an alternative method for cycle time estimation. They start by defining the relationship between cycle time quantiles and system throughput. One nice approach in their method is the comparison to actual data from a case study to show the correlation between real data and estimation data. There are two major assumptions used in their work: the assumption of stationary process convergence, and process mixing, which are based on the theory of queuing system analysis. Process mixing is not relevant for BIW production lines as the series-production setup focuses on a single product, albeit with some minor variations between models.

In their research, Müller et al. 9] assist engineers with a tool to predict robot performance in the packaging industry. Since BIW lines also rely heavily on robots, this research is considered relevant. The method used for robot cycle time estimation is regression analysis. The result is a new software tool, however, it is limited to ABB robot applications only. The motivation for this study originated from a use case, and the main aim is to assist sales engineers with a tool that can give accurate production information about packing robots to potential clients specific to their industry.

There is a lack of research surrounding BIW production lines, and particularly around design topics such as NICT estimation.

\section{$3 \quad$ State of practice}

The current method to estimate NICT for a BIW production line starts by calculating the Takt Time (TT), expressed in seconds;

$$
\mathrm{TT}=\frac{\mathrm{NAT}}{\mathrm{MTT}},
$$

where Mean Throughput Target (MTT) is expressed in units per hour (uph), and Net Available Time (NAT). The NAT is typically assumed to be 3600 seconds per hour.

NICT, in turn, is calculated from talk time and the Overall Equipment Efficiency (OEE) [12;

$$
\begin{aligned}
\mathrm{NICT} & =\frac{O E E \times N A T}{\mathrm{MTT}} \\
& =T T \times O E E .
\end{aligned}
$$

Consider for example a BIW line that is planned to achieve, on average, a throughput of $\mathrm{MTT}=15 \mathrm{uph}$. We use $15 \mathrm{uph}$ as it is aligned with the planned capacity of the line we will use for empirical data later. In the automotive industry an $\mathrm{OEE}=0.85$ is considered best 


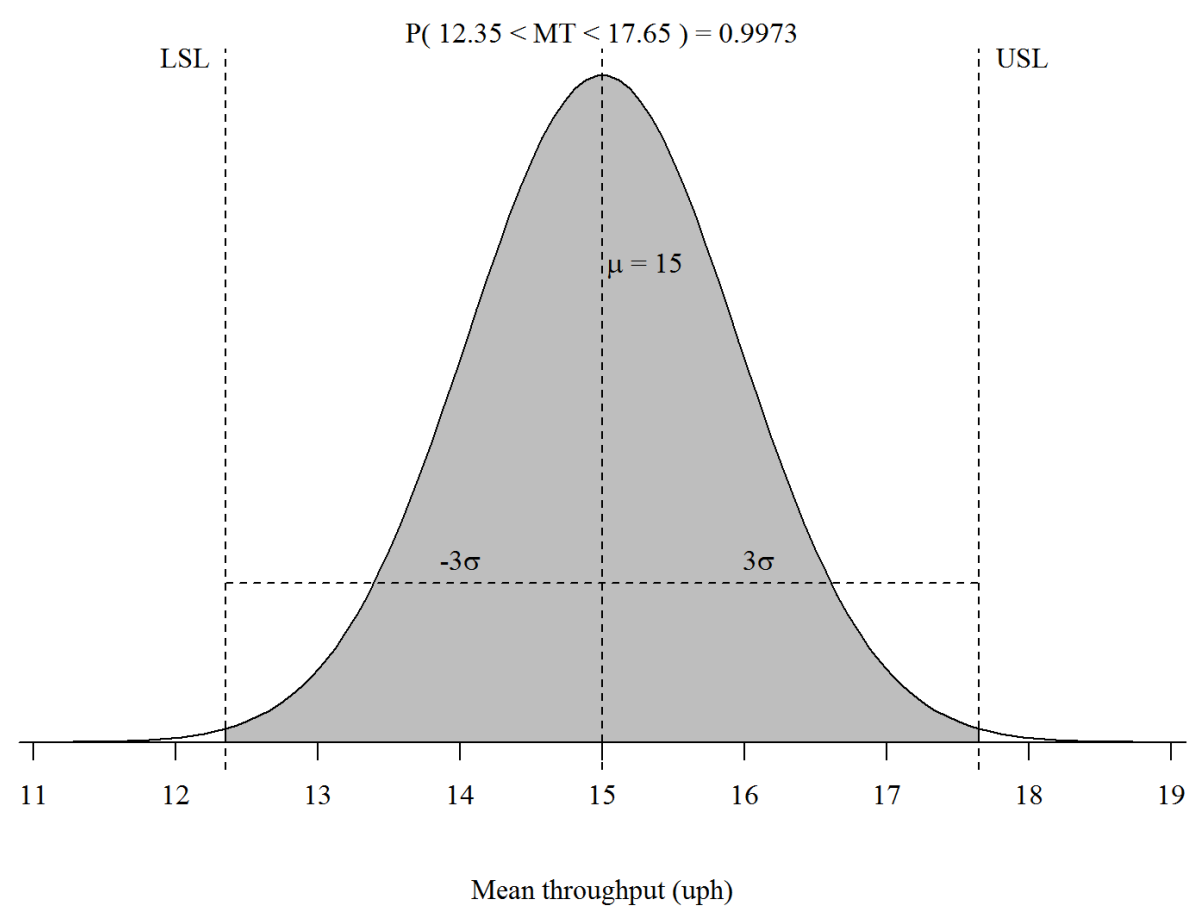

Figure 2: The throughput distribution of a BIW production line fitted into a normal distribution using the three-sigma rule as a starting point.

practice and used in the calculation. The result, when substituting these values into (1) and (2), is an ideal cycle time of

$$
\begin{aligned}
\mathrm{NICT} & =\frac{3600 \times 0.85}{15} \\
& =204 \mathrm{~s},
\end{aligned}
$$

or expressed otherwise, a mean throughput of $17.65 \mathrm{uph}$. This cycle time value is then used as an input into specifying equipment and designing the line capacity.

At the same time line designers account for production variation by subscribing, possibly unconsciously, to the idea that the actual throughput of the production line will follow a normal distribution. More specifically, that under normal production conditions for a line that is under control the average throughput will be within three standard deviations above and below the mean.

$$
P\{\mu-3 \sigma<X<\mu+3 \sigma\}=0.9973 .
$$

This is graphically represented in Figure 2. For this to be true, using the example of a 15 uph line, the standard deviation for actual throughput should be a very ambitious 0.95 uph.

Since the NICT is used to inform equipment decisions, it essentially becomes the throughput ceiling, and we refer to the throughput associated with the NICT as the Upper 


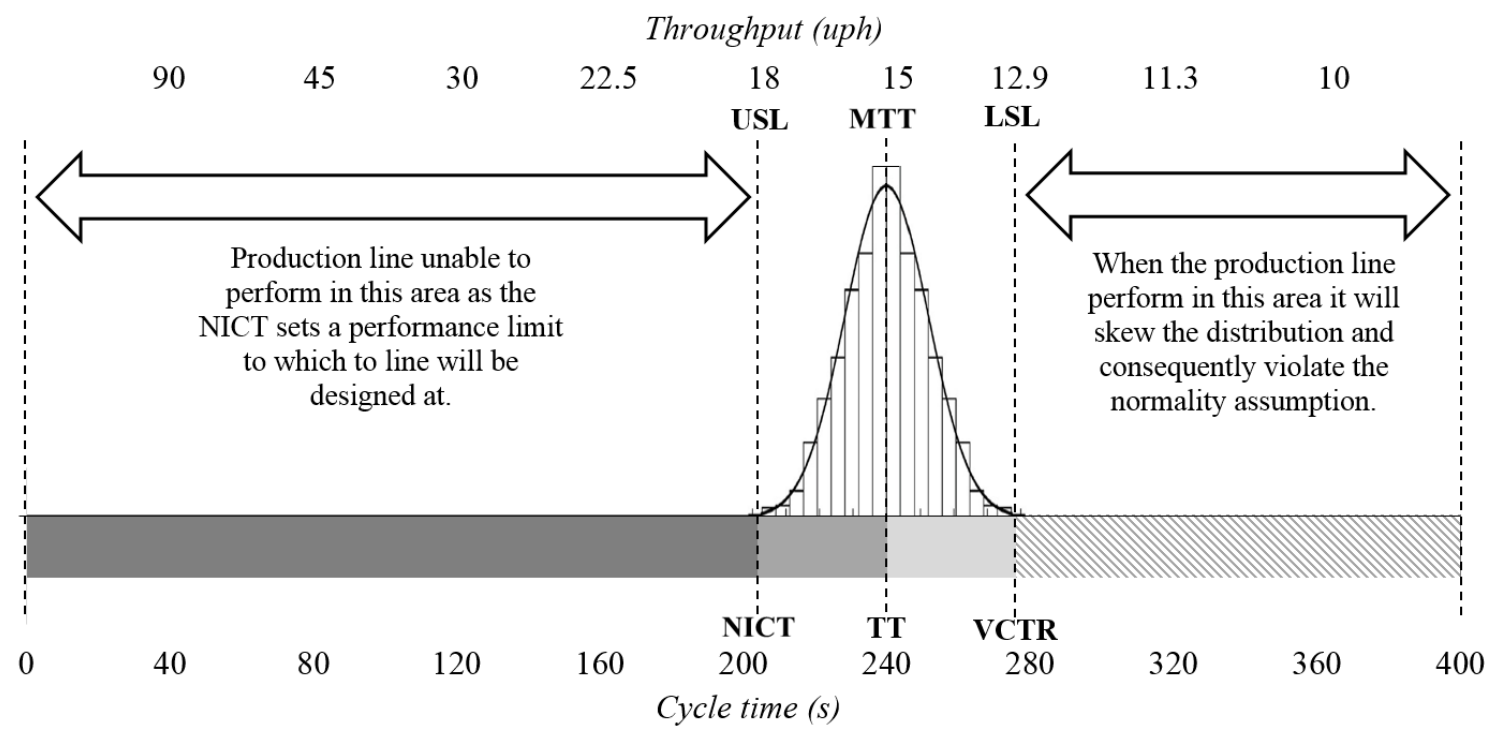

Figure 3: Relationship comparison between throughput and cycle time for a BIW production line with a MTT of 15 units per hour (uph) and a OEE of 0.85 .

Specification Limit (USL). This was calculated earlier as USL $=3600 / 204=17.65$ uph. Similarly, we refer to the throughput that is three standard deviations below the mean, $\mathrm{MTT}=15$, as the Lower Specification Limit (LSL). The cycle time associated with this lower limit is referred to as the Virtual Cycle Time Restriction (VCTR).

The problem with the current NICT estimation can therefore be summarised in Figure 3. Given the equipment limitations, a BIW production line cannot produce more units than the USL as this is the speed for which the line was designed. It also implies that the BIW production line should not produce below the LSL as this will skew the distribution and cause the mean throughput to decrease. In reality, however, breakdowns and labour unrest can easily result in actual throughput being much lower than the LSL.

This intuitive asymmetry around the mean throughput then leads one to question the inherent normality assumption. In the next section we investigate this further using empirical throughput data.

\section{Throughput performance in reality}

We were privy to unrestricted access to all BIW production line information and actual production data for a specific automotive Original Equipment Manufacturer (OEM) on one of their production lines. In this section we describe the methodology to calculate and analyse the mean daily throughput. Data processing and analysis was done with the statistical computing software R [1]. 


\subsection{Methodology}

We extracted data for the period 2012-2015. Every unit that is produced triggers an incremental counter, in real time, in the central database. At the end of each day the date, the number of shifts for that day, the total number of units produced, and the day's volume target is recorded.

From this we calculate the Mean Throughput (MT);

$$
\mathrm{MT}=\frac{\mathrm{TDV}}{\mathrm{DHW}},
$$

where MT is expressed in uph, Total Daily Volume (TDV) is the total daily number of units (volume) that was produced and Daily Hours Worked (DHW) is the total number of hours worked per specific day. With standard 8-hour shifts this would typically just be eight times the number of shifts for the day.

Practical experience and management input suggested that the daily throughput is dependent on the day of the week. There is typically higher absenteeism of employees on Mondays, Fridays and Saturdays, while labour productivity is also lower on these days. Production on Sundays deviates as well, but this is due to operational changes being tested on the production line on Sundays. These daily variations were confirmed in the empirical data and is illustrated in Figure 4.

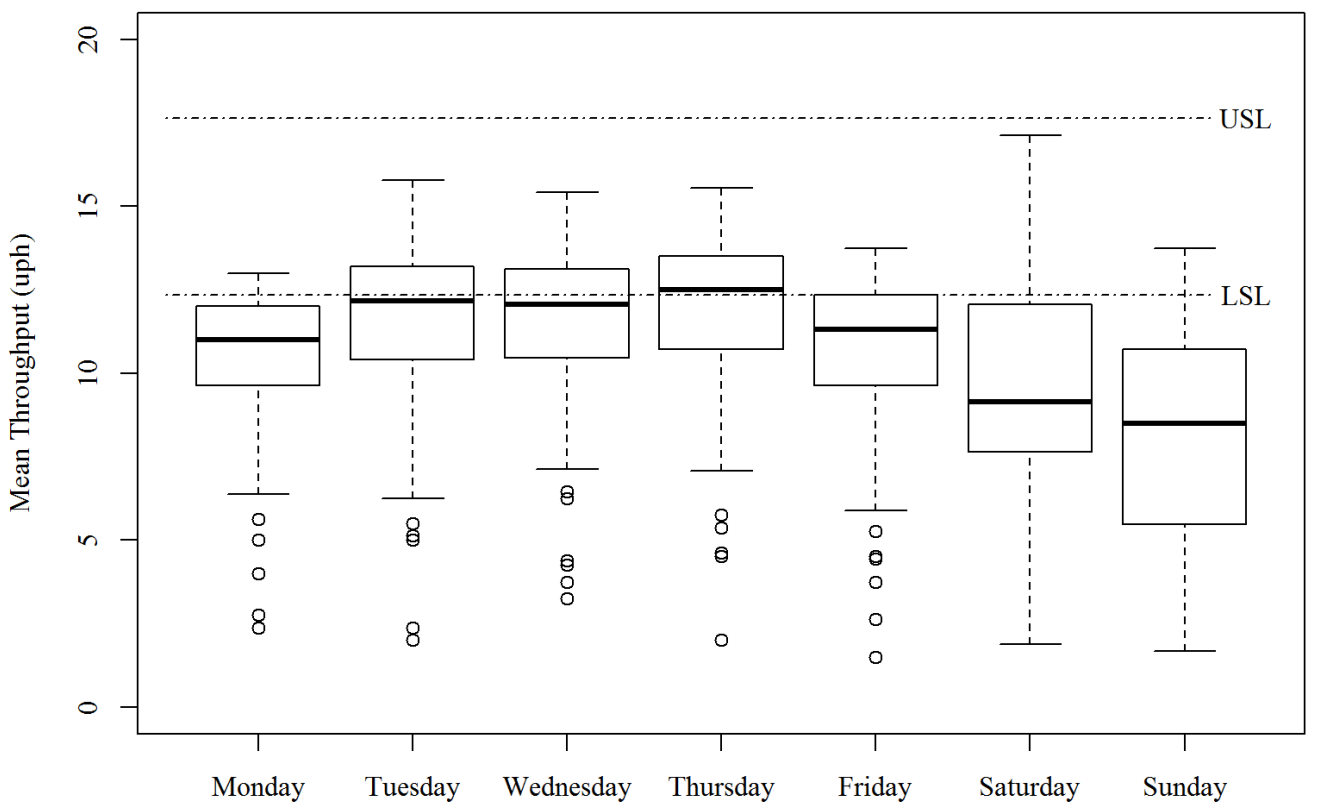

Figure 4: The distribution of daily Mean Throughput (MT) for the years 2012-2015 as a function of the day of the week.

Such variations is, at least partially, under management's control. Since we want to use the throughput data to estimate and inform future design calculations in the NICT, we removed Mondays, Fridays, Saturdays and Sundays. 
The resulting data for the remaining three days were combined. The mean throughput for each production year was plotted in the histograms of Figure 5. The normal distribution

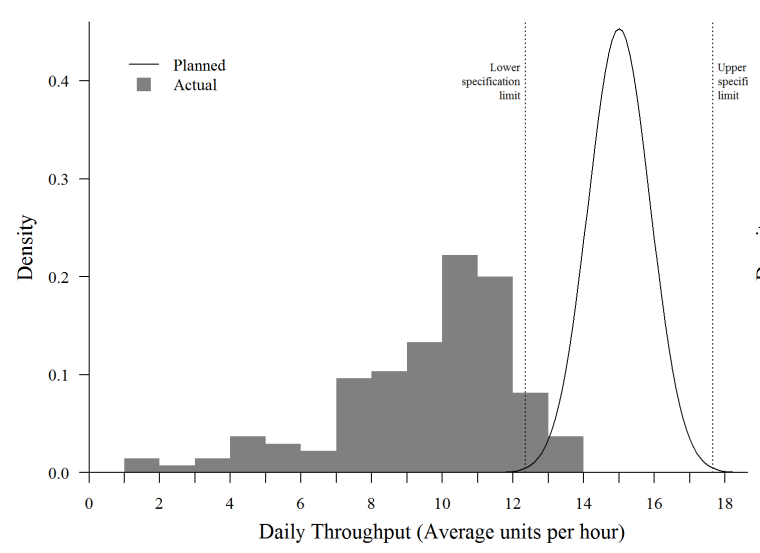

(a) 2012

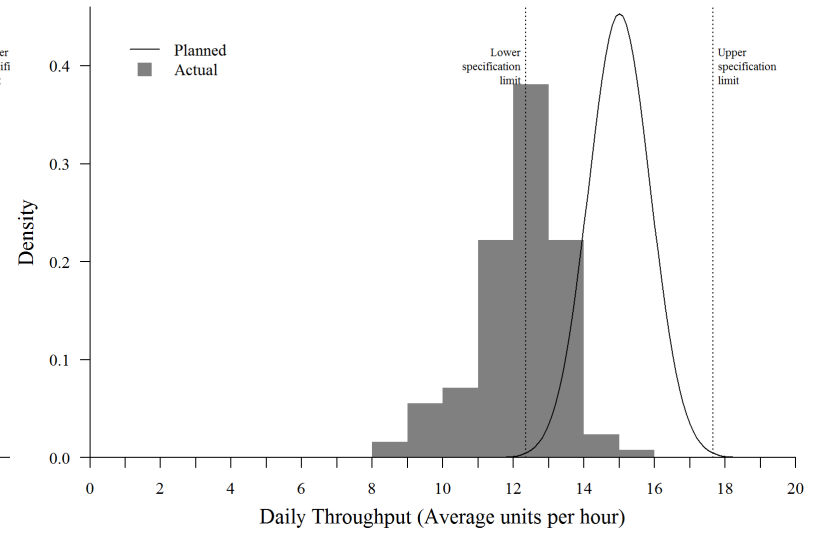

(b) 2013

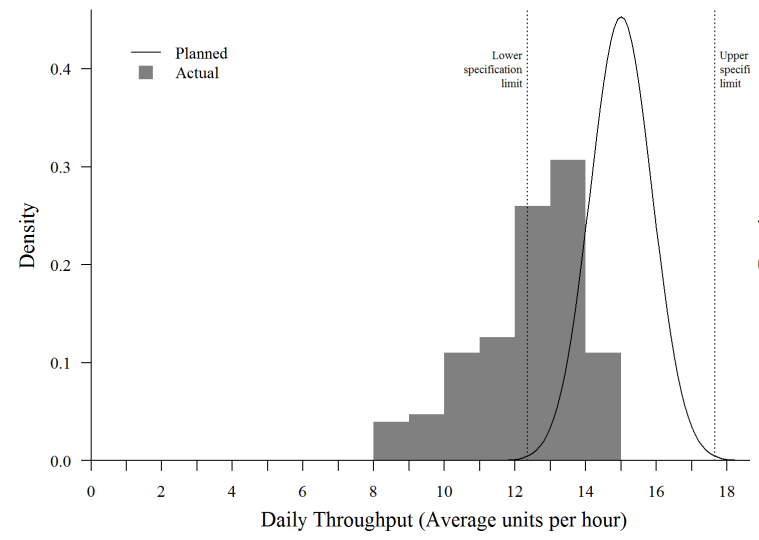

(c) 2014

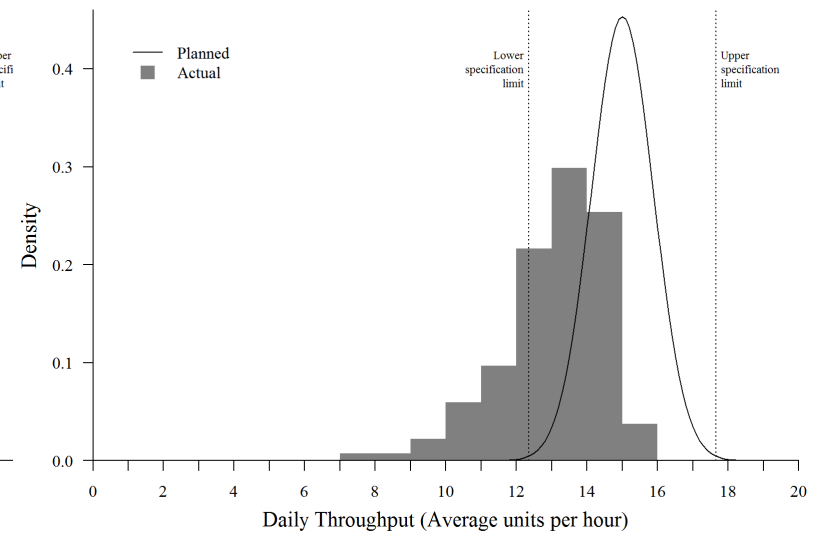

(d) 2015

Figure 5: The empirical Mean Throughput (MT) distribution, expressed in uph, for the period 2012-2015.

in each histogram represents a comparative reference to the planned throughput for a line of the same capacity, $15 \mathrm{uph}$. The assumption of the comparative reference is in line with our earlier discussion of the state of practice: a) actual output would be within three standard deviations of the MTT of 15 uph; and b) the standard deviation for the line is $\sigma=0.95$ uph.

Three observations are worth making at this point. Firstly, we see that the empirical data was never distributed within the limits of the LSL and USL. Secondly, the throughput distribution does not appear to be normally distributed, and is consistently left-skewed. This is in line with the earlier argument that the USL acts as a production ceiling, while lower-than-limit production is indeed possible. Thirdly, there is a noticable difference between 2012 and the other years in terms of the long left tail. This performance 
difference is a result of 2012 being the launch year of a new product. In the launch year performance is gradually increased from a very low throughput base. It usually takes the BIW production line about six months from start of production to mature before the line reaches its maximum performance.

\subsection{Empirical throughput performance}

To identify an appropriate distribution we start by giving some summary statistics about each year's throughput data in Table 1 .

\begin{tabular}{lrrrrrr}
\hline & \multicolumn{7}{c}{ Year } & & & \\
Statistic & $\mathbf{2 0 1 2}$ & $\mathbf{2 0 1 3}$ & $\mathbf{2 0 1 4}$ & $\mathbf{2 0 1 5}$ & Mean & SD \\
\hline Min & 2.00 & 8.17 & 8.42 & 7.21 & 6.45 & 2.61 \\
Median & 10.33 & 12.27 & 12.83 & 13.29 & 12.18 & 1.13 \\
Mean & 9.64 & 12.19 & 12.40 & 13.08 & 11.83 & 1.31 \\
Max & 13.75 & 15.38 & 14.63 & 15.79 & 14.89 & 0.78 \\
Standard deviation & 2.517 & 1.267 & 1.503 & 1.502 & 1.697 & 0.483 \\
Skewness & -1.034 & -0.650 & -0.740 & -1.109 & -0.883 & 0.193 \\
Kurtosis & 3.829 & 3.759 & 2.799 & 4.586 & 3.743 & 0.634 \\
\hline
\end{tabular}

Table 1: Summary statistics for the empirical throughput: 2012-2015.

The actual standard deviation, for each year, is much higher that the required $\sigma=0.95$ for the values to fall within three standard deviations around the mean.

The sample skewness is a measure of the data's asymmetry about its mean. Negative values is indicative of a skewed distribution. In all cases the value is significantly different from zero, confirming that the data is not symmetric around the mean. The kurtosis metric is a measure of the heavy-tailedness of the distribution of the data. Higher kurtosis means more of the variance is the result of infrequent extreme observations, as opposed to more frequent but modestly sized deviations. A kurtosis value of less than 3 (2014) suggests that the distribution is platykurtic, meaning there are fewer and less extreme outliers than what one might find in a normal distribution with the same mean and standard deviation. In a quality assurance sense, this could be indicative of a production line that is 'under control'. For the other three years the kurtosis values exceed 3 suggesting the distributions are leptokurtic and contain more outliers than one would find in a corresponding normal distribution.

We used the fitdistrplus package in $\mathrm{R}$ [4] and prepared a Cullen and Frey graph to identify other possible distributions that could potentially fit the empirical data better. For a number of common distributions, the graph indicates the range of skewness and kurtosis values associated with that distribution. However, because skewness and kurtosis are not robust statistics, we take the uncertainty of the estimated values of kurtosis and skewness into account. A non-parametric bootstrap procedure, based on Efron \& Tibshirani [6], is performed. Values of skewness and kurtosis are computed on bootstrap samples: drawing random samples with replacement from the original data set. The resulting estimates for the 2015 data set is given in Figure 6.

The Cullen and Frey reference graph consists out of points, lines and surfaces. According 


\section{Cullen and Frey graph}

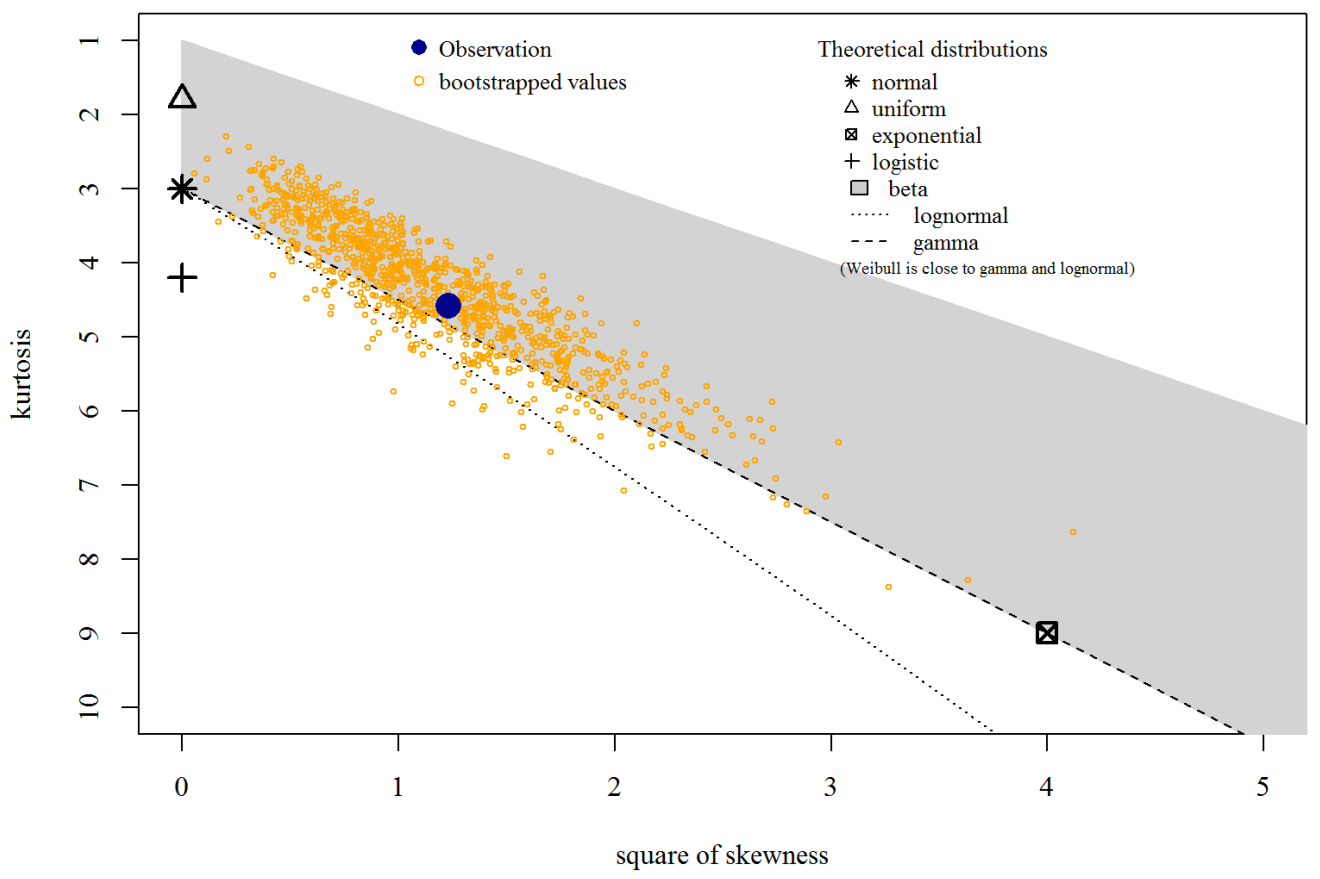

Figure 6: The 2015 throughput data of the reference BIW plotted as a Cullen and Frey graph. Produced using [4, 11].

to Delignette-Muller \& Dutang [4, distributions like the normal, uniform, logistic and exponential can have only one skewness and kurtosis value, and are therefore represented by a single point. For distributions like the log-normal and gamma, more values are possible and these distributions can fall anywhere on the given line. For distributions like the beta distribution there is an entire surface of values within which the distribution can lie.

Our empirical dataset for 2015 is represented by both the single estimate, as well as the bootstrap samples. These values coincide with that of a beta, log-normal, gamma and/or Weibull distribution. The data for years 2012-2014 yield similar results.

We calculated several measures of fit for the various distributions - gamma, normal, Weibull, log-normal, exponential and logistic - to see which one matches with our data best. All distribution results were compared with one another in terms of Log likelihood (LL), Akaike's Information Criterion (AIC) and Bayesian Information Criterion (BIC), and are displayed in Table 2, Low values imply a better fit. The Weibull distribution consistently performed best in these comparisons. A visual confirmation of the fit is seen in a number of statistical plots illustrated in Figure 7.

Consider the Quantile-Quantile (Q-Q) plot of Figure 7b. The fitted Weibull distribution describes the scale and shape of the dataset well. Although the tail behaviour is similar, the fitted Weibull slightly over-predicts the lower throughput values. This may actually 


\begin{tabular}{|c|c|c|c|c|c|c|c|c|c|c|c|c|}
\hline & \multicolumn{3}{|c|}{2012} & \multicolumn{3}{|c|}{2013} & \multicolumn{3}{|c|}{2014} & \multicolumn{3}{|c|}{2015} \\
\hline & LL & AIC & BIC & LL & AIC & BIC & LL & AIC & BIC & LL & AIC & BIC \\
\hline Weibull & -313 & 630 & 636 & -206 & 416 & 421 & -224 & 451 & 457 & -233 & 469 & 475 \\
\hline Logistic & -314 & 631 & 637 & -207 & 417 & 423 & -233 & 470 & 476 & -241 & 486 & 492 \\
\hline Normal & -320 & 644 & 650 & -210 & 424 & 430 & -233 & 469 & 475 & -248 & 501 & 507 \\
\hline Gamma & -362 & 727 & 733 & -216 & 435 & 441 & -238 & 481 & 486 & -261 & 525 & 531 \\
\hline Log-normal & -402 & 808 & 814 & -219 & 443 & 448 & -242 & 488 & 494 & -268 & 541 & 547 \\
\hline Exponential & -446 & 895 & 898 & -448 & 897 & 900 & -453 & 908 & 911 & -485 & 973 & 976 \\
\hline
\end{tabular}

Table 2: Distribution fit results for all data (2012-2015) using various test distributions.

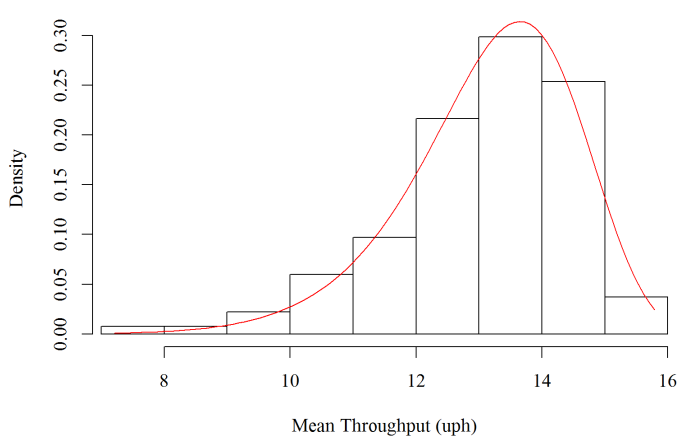

(a) Histogram and theoretical densities

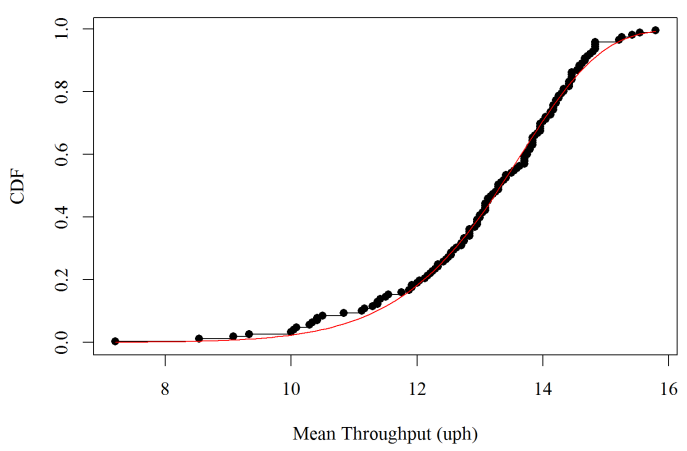

(c) Empirical and theoretical CDFs

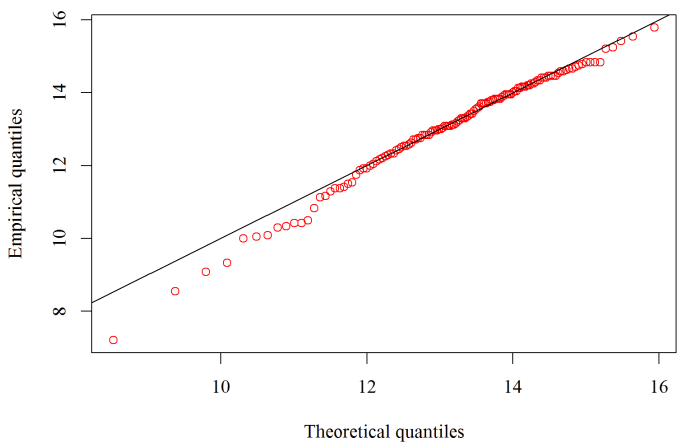

(b) Q-Q plot

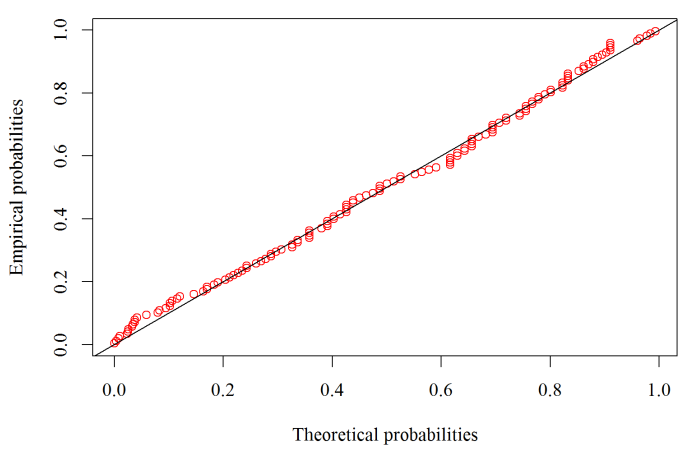

(d) P-P plot

Figure 7: The 2015 throughput data of the reference BIW compared to a theoretical throughput of a Weibull distribution.

be to our benefit as it means we will provide a conservative estimate for the NICT. The over-prediction of low-throughput instances is confirmed in the higher probabilities in the Probability-Probability (P-P) plot of Figure $7 \mathrm{~d}$

The current NICT estimation method does not make an explicit assumption that the MT of a BIW production line is normally distributed, but it assumes that the performance characteristics of all production lines are similar. As a result, the current estimator ignores any possible past performance of a production line, or industry experience. Instead, we can now propose an estimator that uses our knowledge of past performance. 


\section{Estimating NICT using a Weibull distribution}

A Weibull distribution is described using two parameters: shape $(k)$ and scale $(\lambda)$. The empirical data gives us a good idea of what the shape parameter must be. The scale, however, can be left as an input parameter for the designer to determine the NICT so that the actual MTT can be achieved.

The shape of the distribution can be estimated by taking the mean shape for all the empirical years, excluding 2012. The data from 2012 was omitted since the launch-year is a valid assignable cause. By omitting 2012's shape parameter, the mean shape is increased from $\bar{k} \approx 9.58$ to $\bar{k} \approx 11.17$, and at the same time the standard deviation of these estimates is reduced from $k_{\sigma} \approx 2.77$ to $k_{\sigma} \approx 0.37$.

Given the mean, $\mu$, the Weibull distribution's scale parameter, $\lambda$, can be calculated using the relation

$$
\mu=\lambda \Gamma(1+1 / k)
$$

where $\Gamma$ is the gamma function and $k$ is the (estimated) shape parameter, $\bar{k}$, of the Weibull distribution, see [17. The gamma function is an extension of the factorial function and can be expressed as

$$
\Gamma(x)=\int_{0}^{\infty} z^{x-1} e^{-z} d z,
$$

where $x$ is positive. The estimated standard deviation for the Weibull distribution, $\sigma$, can be calculated using

$$
\sigma^{2}=\lambda^{2}\left[\Gamma(1+2 / k)-\Gamma(1+1 / k)^{2}\right] .
$$

The calculated standard deviation can be compared to the empirical data reported in Table 1. The USL is related to the quantile function, or the inverse cumulative distribution;

$$
\mathrm{USL}=\lambda[-\ln (1-p)]^{\frac{1}{k}} .
$$

For a given probability $p$ the quantile function provides a value (mean throughput in this case) such that the random variable (throughput) is less than or equal to this value with probability $p$. Knowing $p$ we can calculate the NICT;

$$
N I C T=\frac{N A T}{\lambda[-\ln (1-p)]^{\frac{1}{k}}},
$$

where $N A T$ is again $3600 \mathrm{~s}$. The value of $p$ could be considered the required certainty with which BIW line designers want to estimate the NICT. Consider the earlier discussion where line designers assume the three-sigma rule, $p=0.9973$. That is, $99.73 \%$ of all observations should fall below the USL. 


\section{Discussion of results}

In this section we apply our proposed Weibull-based method to the example line with a MTT of $15 \mathrm{uph}$. From the empirical data of 2013-2015 we use a shape parameter of $\bar{k}=11.17$. Using (4) we calculate the scale parameter as $\lambda=15.53$, and using (5) we calculate $\sigma=1.61$. This is quite close the the mean empirical standard deviation of $\sigma=1.69$.

The final step is estimating that NICT $\approx 197.67 \mathrm{~s}$ and the associated USL $\approx 18.21 \mathrm{uph}$. This is a $3.2 \%$ increase in throughput over the NICT when using the current, traditional method. Consequently, one can expected an approximate increase of $3 \%$ in financial investment as well, to account for higher capacity equipment requirements.

A total of 10 different BIW investment projects were analysed to determine an average investment figure as well as an average loss per unit from the production program. This information was again supplied by the reference OEM. In the following example we scaled the real figures to protect the confidentiality of investment and loses figures of the specific OEM.

Consider a new BIW production line where 3.25 Million Euros are required per unit per hour to achieve the required MTT. For every one unit that is lost from the production program, there is a 2,000 Euro income loss for the specific production facility. This can quickly result in a negative business case if the daily income is surpassed by the daily fixed and running costs. This means that for a BIW production line with a planned MTT of $15 \mathrm{uph}$, the required investment would be 48.75 Million Euro using the current method, and 50.26 Million Euro for our proposed method. This is an increase of 1.51 Million Euro, or approximately $3.1 \%$.

Over the 7 year production lifecycle, and using the empirical data, we can calculate that the expected losses would total 1646 units due to unforeseen process variations that are not catered for by the current NICT estimation method. The 1646 units loss equates to a monetary loss of 3.24 Million Euro over the production life cycle.

This means that using the proposed method instead of the current method, the total loss can be reduced by almost $50 \%$ in monetary terms. The proposed method covers in our case four years of real production history. Included in this history is unforeseen events such as strikes, major power outages and various other variantions that are not consider when defining NICT. The Weibull estimate honours the production ceiling, as well as the left-skewed distribution observed in empirical data.

\section{Conclusion}

Analysing empirical data of a real BIW production line, we can conclude that the mean daily throughput follows a Weibull distribution. We propose a new method to estimate NICT based on this distribution.

Our proposed method has a number of advantages over the current method used for estimating NICT. Firstly, the Weibull-based method is more representative of the real 
production characteristics and behaviour of the automotive BIW production lines. This allows for more realistic and reliable planning. However, this approach requires historical production data for the specific line considered or a similar line.

Secondly, knowing the Weibull distribution parameters allow a more vivid capability target for performance measurement and control. Our new method resulted in a $3.1 \%$ increase in estimated NICT, which will be different for every BIW production line.

In the illustrative example we noted a $3.2 \%$ increase in the estimated cycle time, which would equate to a probable investment increase of a similar magnitude to compensate for the faster production line. The increase in investment can be justified (or rejected) by comparing it to the expected losses due to variation of the relevant BIW production line, and it is proposed that a simple business case can determine if the extra financial investment outweighs the expected production losses.

Our study focused purely on one specific BIW production line where only one variant is produced. There are production lines where two or even three automotive product variants are produced. These lines are more complex but should yield similar results. It is proposed to not only investigate these multiple variant production lines, but also production lines where higher throughputs are applicable, for example 45 uph lines. It would be engrossing to see what shape and scale other BIW production lines exhibit, and if there are any shape or scale correlations between these different throughput lines. Further research is proposed with focus on other manufacturing industries where NICT is also used as a production line design input.

\section{References}

[1] Akhavan-Tabatabaei R, Ding S \& Shanthikumar JG, 2009, A method for cycle time estimation of semiconductor manufacturing toolsets with correlations, Proceedings of the 2009 Winter Simulation Conference (WSC), Austin (TX), pp. 1719-1729.

[2] Akhavan-Tabatabaei R, Ucros JJ \& Shanthikumar GJ, 2010, Application of Erlang distribution in cycle time estimation of toolsets with WIP-Dependant arrival and service in a single product-type single failure-type environment, Proceedings of the 2010 Winter Simulation Conference, Baltimore (MD), pp. 2531-2540.

[3] Chen N \& Zhou S, 2011, Simulation-based estimation of cycle time using quantile regression, IIE Transactions, 43(3), pp. 176-191.

[4] Delignette-Muller Ml \& Dutang C, 2015, fitdistrplus: An R Package for fitting distributions, Journal of Statistical Software, 64(4), pp. 1-34.

[5] Delp D, Si J \& Fowler JW, 2006, The development of the complete X-Factor contribution measurement for improving cycle time and cycle time variability, IEEE Transactions on Semiconductor Manufacturing, 19(3), pp. 352-362.

[6] Efron B \& Tibshirani RJ, 1994, An Introduction to the Bootstrap, CRC Press, Boca Raton (FL).

[7] Huang HP, Yeh CF, Juang JY, Lin LR \& Chen T, 1998, Dynamic average method for cycle time estimator in an IC fab, 1998 Semiconductor Manufacturing Technology Workshop, Hsinchu.

[8] Johri PK, 1987, A linear programming approach to capacity estimation of automated production lines with finite buffers, International Journal of Production Research, 25(6), pp. 851-866. 
[9] Müller M, Kuhlenkötter B \& Geiger H, 2014, Cycle time estimation for a delta-type robot, $41^{\text {st }}$ Symposium on Robotics, Munich, pp. 225-231.

[10] Pearn WL, Tai YT \& LeE JH, 2009, Statistical approach for cycle time estimation in semiconductor packaging factories, IEEE Transactions on Electronics Packaging Manufacturing, 32(3), pp. 198-205.

[11] R Development Core Team, 2016, R: A language and environment for statistical computing.

[12] Stamatis DH, 2010, The OEE primer - Understanding Overall Equipment Effectiveness, CRC Press, New York (NY).

[13] TAI YT, PEARN WL \& LeE JH, 2012, Cycle time estimation for semiconductor final testing processes with Weibull-distributed waiting time, International Journal of Production Research, 50(2), pp. $581-592$.

[14] Veeger CPL, Etman LFP, Van Herk J \& Rooda JE, 2009, Predicting the mean cycle time as a function of throughput and product mix for cluster tool workstations using EPT-based aggregate modeling, pp. 80-85.

[15] Xu M, Abdul-Kader W \& Ganjavi O, 2009, Cycle time estimation of a multiple product production line: An approach with fuzzy and chance-constrained programming, Infor, 47(2), pp. 93-103.

[16] Yang F, Ankenman BE \& Nelson BL, 2005, Estimation of percentiles of cycle time in manufacturing simulation. Proceedings of the 2005 Winter Simulation Conference (WSC), Orlando (FL), pp. 475-484

[17] Yang G, 2007, Life Cycle Reliability Engineering, John Wiley \& Sons, New York (NY).

[18] Zhou Z \& Rose O, 2013, Cycle time variance minimization for WIP balance approaches in wafer fabs, Proceedings of the 2013 Winter Simulation Conference, Washington (DC), pp. 3777-3788. 